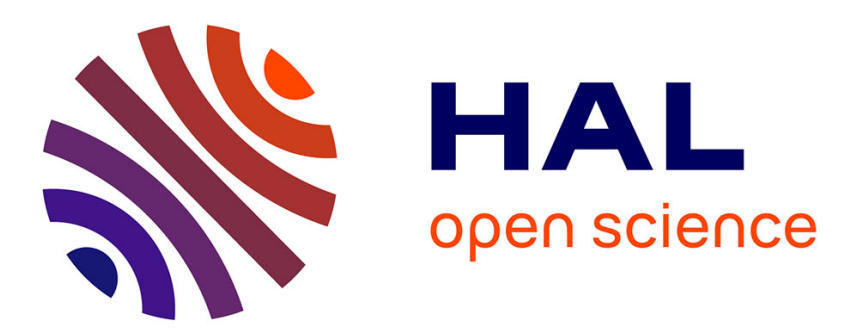

\title{
More knocks to the oxidation hypothesis for vascular disease?
}

\author{
Adam Mullan, Naveed Sattar
}

\section{To cite this version:}

Adam Mullan, Naveed Sattar. More knocks to the oxidation hypothesis for vascular disease?. Clinical Science, 2008, 116 (1), pp.41-43. 10.1042/CS20080486 . hal-00479450

\section{HAL Id: hal-00479450 https://hal.science/hal-00479450}

Submitted on 30 Apr 2010

HAL is a multi-disciplinary open access archive for the deposit and dissemination of scientific research documents, whether they are published or not. The documents may come from teaching and research institutions in France or abroad, or from public or private research centers.
L'archive ouverte pluridisciplinaire HAL, est destinée au dépôt et à la diffusion de documents scientifiques de niveau recherche, publiés ou non, émanant des établissements d'enseignement et de recherche français ou étrangers, des laboratoires publics ou privés. 
More knocks to the oxidation hypothesis for vascular disease?

\section{Adam Mullan, Naveed Sattar}

British Heart Foundation Glasgow Cardiovascular Research Centre Faculty of Medicine, University of Glasgow 126 University Place, Glasgow G12 8TA

\section{Address for Correspondence:}

Dr Adam Mullan or Professor Naveed Sattar BHF GCRC

Faculty of Medicine University of Glasgow

Phone: $+44(0) 1412114312$

Fax: $\quad+44(0) 1415532558$

Email:

adamwfmullan@yahoo.co.uk nsattar@clinmed.gla.ac.uk 


\begin{abstract}
The oxidation hypothesis for coronary heart disease (CHD) is attractive.

However, the almost universal failure of antioxidant vitamin supplementation as a CVD risk modifier challenges the oxidation hypothesis, at least as a concept that easily 'translates' into clinical benefit for the population. At the same time, quality prospective data on lipid or protein oxidation markers as predictors of vascular events is spare. In this issue of the journal, Woodward and colleagues provide much needed prospective data examining the relationship between markers of oxidative damage and CHD outcome in a general population. Despite noting the expected associations between several established CHD risk factors and CHD events, no significant link was seen between measured oxidation markers and $\mathrm{CHD}$ risk, a finding which further challenges the oxidation hypothesis for CHD.
\end{abstract}


Healthy human physiology relies on a complex balance of pro- and antioxidant mechanisms. Reactive oxygen species such as superoxide anions, free radicals and hydrogen peroxide, are generated as by-products of aerobic metabolism. These prooxidant systems are balanced by a battery of antioxidants such as exogenously derived ascorbic acid and endogenous superoxide dismutase. Pro-oxidant effects are essential for normal processes involved in cell apoptosis and innate immune responses to infection. When oxidative stress occurs in excess and overwhelms anti-oxidant reserves, lipids, proteins and DNA are indiscriminately damaged, often rendering them dysfunctional and contributing to the development of chronic disease.

A central role for oxidative stress in cardiovascular disease (CVD) is an attractive theory, particularly given that antioxidant administration (in the form of 'simple and safe' vitamin supplements) might reverse or at least slow this process in some way. Prior consistent epidemiological evidence of low vitamin levels in subjects at risk of CVD has fuelled an explosion of end-point trials investigating the cardio-protective potential of vitamin supplements. Antioxidant supplementation has, however, generally failed to demonstrate any beneficial effect. A recent Cochrane meta-analysis of 77 randomised trials involving 232,550 participants concluded that there was no evidence to support the use of antioxidants in primary or secondary CVD prevention and, in fact, suggested a potentially deleterious effect with vitamin A, beta-carotene and Vitamin E [1]. Similarly, observational studies have linked circulating homocysteine levels with the risk of CVD; however despite successfully lowering homocysteine levels, interventional studies of folic acid and cyanocobalamin have not shown any reduction in CVD risk[2;3]. This illustrates an important concept: attempting to reverse the pathophysiological mechanisms linked to apparently aberrant biomarker levels (in this case low antioxidant levels) does not necessarily translate into improved outcomes.

The almost universal failure of antioxidant vitamin supplementation as a CVD risk modifier certainly threatens the oxidation hypothesis, at least as a concept that translates into clinical benefit for the population. However, a number of considerations have been proposed in this area to try to explain past failures and to help improve future research. The Cochrane review suggests that trial methodologies in the antioxidant field have generally been suboptimal: patient groups varied dramatically with no estimation of baseline oxidative stress levels; there was uncertainty regarding baseline and cardiovascular health at outcome; and risk of bias was high. The interventional supplements also varied massively in terms of dose, duration of treatment, and combinations used. Of course, the results of the antioxidant randomised controlled trials (RCTs) have to be taken at face value; there is currently no role for supplements of naturally occurring antioxidants in the prevention of CVD. With this in mind, it is of interest that Lawlor and colleagues recently showed that socio-economic factors may confound the relationship between low vitamin levels and CVD risk[4]. In other words, low vitamin levels may not be causally linked to CVD risk; rather they are simply markers of some other more relevant process also linked to socio-economic status.

With the above body of evidence in mind, there is clearly a need for parallel prospective studies linking oxidative stress markers measured at baseline to incident CVD outcomes. But which markers are best and how easy are they to measure? An ever increasing number of biomarkers of oxidative stress are emerging, relying on identification of invivo lipid and protein targets for oxidation (reviewed[5;6]). F2-Isoprostanes, derived 
from non-enzymatic free-radical mediated peroxidation of arachidoic acid, are arguably the best established biomarker of oxidative stress. In relation to CVD, they have been found to be significantly increased in atherosclerotic plaques compared to healthy nonatherosclerotic vascular tissue; systemic F2-Isoprostanes have also been found to increase following angioplasty. The relationship between oxidized LDL-cholesterol (oxLDL) and CVD has also been extensively studied, and there is some evidence, albeit mixed, for oxLDL markers as in-vivo markers of oxidative stress. Unregulated uptake of oxLDL by scavenger receptors on macrophages results in the formation of foam cells, precursors of advanced atheromatous plaque formation. OxLDL is also thought to i) enhance interactions between monocytes and endothelial cells to promote atherogenesis and plaque instability, ii) induce the renin-angiotensin system, and iii) promote a cascade of inflammatory cytokines such as IL-6, and TNF-alpha. Finally, HDL particles are another target for oxidative stress, and it is possible to measure oxidised apolipoproteins (apo) AI and A-II as markers for in-vivo HDL-cholesterol oxidation. The role of oxidised HDL (oxHDL) in atherogeneis is however less clear. Studies have suggested that oxidation of methionine residues on apoA-I impairs reverse cholesterol transport; however studies in mice suggest that tyrosyl radical oxHDL may be more effective at extracting cholesterol[7].

Measurement of markers of oxidative stress is therefore complex and also requires careful sample collection and storage, and invariably labour intensive methodology, such as high pressure liquid chromatography (HPLC) for oxHDL or mass spectrometry for F2-isoprostanes, as described by Woodward et al [8]. Other methods involve immunoassays to oxidised moieties of oxLDL. However there are lingering concerns over specificity of some ELISA based methods as well as uncertainty about the pathophysiological relevance of some oxidative markers measured in the circulation versus tissue levels (where levels may change in opposite directions). Accurate assessment or interpretation of oxidative stress markers in large scale population based studies is therefore not easy and, as a result, there are few robust studies prospectively linking oxidative biomarkers with CVD incident end-points. Small-scale studies have suggested a role for F2-Isoprostanes (measured in plasma or urine) as independent risk markers for atherosclerosis [9;10]; however larger prospective studies, with proper adjustment for confounding factors, are noticeably sparse [6].

In the current issue of the journal, Woodward et. al report a new prospective study in this area. They measured F2-Isoprostanes, and oxidised moieties on apoliprotein AI and AII, HDL-related proteins, in a nested case control study with 227 coronary cases and 420 controls. Woodward et al. noted no significant association between measured markers and CVD event risk. Whether this study had sub-optimal power to detect associations between oxidation markers and CHD risk is debatable since established risk predictors were significantly related to outcomes. In short, the results are disappointing with regards to the oxidation hypothesis. There is little other data on oxHDL moieties available for comparison but other markers of oxidations, for example oxidised phospholipids /apoB measurement (an antibody based assay) has been shown by others to be predictive of 10year CVD events in a prospective population based study of 765 subjects[11]. To advance this field, careful collaboration between different groups is required to generate greater power in prospective studies of a range of oxidative markers. Such studies should also employ, wherever possible, gold standard or at least standardised laboratory methods to measure oxidative stress so that results are more robust. 
In summary, although the oxidation hypothesis for vascular disease is attractive, there is currently inconsistent evidence linking markers of oxidative stress with incident CVD events. Equally, RCTs of vitamins have failed to show vascular benefit and in some cases have suggested possible harm. That said, a recent RCT with a synthetic antioxidant, succinobucol[12], did show some promise in terms of vascular and diabetes risk reduction although the trial failed to meet its primary end-point and reported some unexpected harms. Given these uncertainties, the foundations of CVD risk prediction should continue with established risk factors such as lipids, blood pressure, and smoking to determine risk and proven interventions to lessen it. 
Reference

1. Bjelakovic,G., Nikolova,D., Gluud,L.L., Simonetti,R.G. and Gluud,C. (2008) Antioxidant supplements for prevention of mortality in healthy participants and patients with various diseases. Cochrane.Database.Syst.Rev. CD007176.

2. Bazzano,L.A., Reynolds,K., Holder,K.N. and He,J. (2006) Effect of folic acid supplementation on risk of cardiovascular diseases: a meta-analysis of randomized controlled trials. JAMA 296, 2720-2726.

3. Ebbing,M., Bleie,O., Ueland,P.M. et al. (2008) Mortality and cardiovascular events in patients treated with homocysteine-lowering B vitamins after coronary angiography: a randomized controlled trial. JAMA 300, 795-804.

4. Lawlor,D.A., Davey,S.G., Kundu,D., Bruckdorfer,K.R. and Ebrahim,S. (2004) Those confounded vitamins: what can we learn from the differences between observational versus randomised trial evidence? Lancet 363, 1724-1727.

5. Tsimikas,S. (2008) In vivo markers of oxidative stress and therapeutic interventions. Am.J.Cardiol. 101, 34D-42D.

6. Tsimikas,S., Willerson,J.T. and Ridker,P.M. (2006) C-reactive protein and other emerging blood biomarkers to optimize risk stratification of vulnerable patients. J.Am.Coll.Cardiol. 47, C19-C31.

7. Macdonald,D.L., Terry,T.L., Agellon,L.B., Nation,P.N. and Francis,G.A. (2003) Administration of tyrosyl radical-oxidized HDL inhibits the development of atherosclerosis in apolipoprotein E-deficient mice. Arterioscler.Thromb.Vasc.Biol. 23, 1583-1588.

8. Woodward,M., Croft,K.D., Mori,T.A. et al. (2008) The association between both lipid and protein oxidation and the risk of fatal or non-fatal coronary heart disease in a human population. Clin.Sci.(Lond).

9. Vassalle,C., Botto,N., Andreassi,M.G., Berti,S. and Biagini,A. (2003) Evidence for enhanced 8-isoprostane plasma levels, as index of oxidative stress in vivo, in patients with coronary artery disease. Coron.Artery Dis. 14, 213-218.

10. Schwedhelm,E., Bartling,A., Lenzen,H. et al. (2004) Urinary 8-iso-prostaglandin F2alpha as a risk marker in patients with coronary heart disease: a matched casecontrol study. Circulation 109, 843-848.

11. Kiechl,S., Willeit,J., Mayr,M. et al. (2007) Oxidized phospholipids, lipoprotein(a), lipoprotein-associated phospholipase A2 activity, and 10-year cardiovascular outcomes: prospective results from the Bruneck study.

Arterioscler.Thromb.Vasc.Biol. 27, 1788-1795. 
12. Tardif,J.C., McMurray,J.J., Klug,E. et al. (2008) Effects of succinobucol (AGI1067) after an acute coronary syndrome: a randomised, double-blind, placebocontrolled trial. Lancet 371, 1761-1768. 